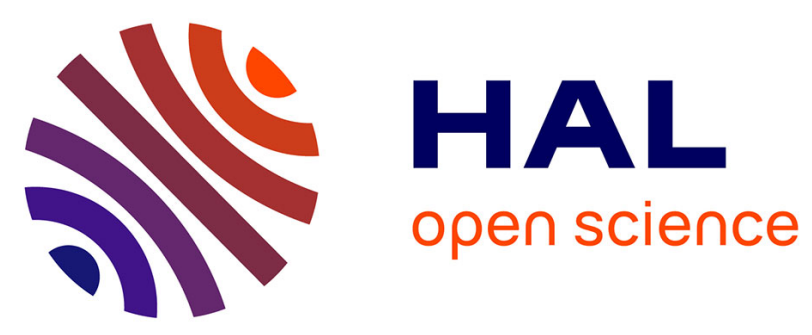

\title{
PERFORMANCE EVALUATION OF POINT MATCHING METHODS IN VIDEO SEQUENCES WITH ABRUPT MOTIONS
}

\author{
Wael Elloumi, Sylvie Treuillet, Rémy Leconge, Aïcha Fonte
}

\section{To cite this version:}

Wael Elloumi, Sylvie Treuillet, Rémy Leconge, Aïcha Fonte. PERFORMANCE EVALUATION OF POINT MATCHING METHODS IN VIDEO SEQUENCES WITH ABRUPT MOTIONS. VISAPP, Fifth International Conference on Computer Vision Theory and Applications, May 2010, Angers, France. pp.427-430. hal-00678782

\section{HAL Id: hal-00678782 \\ https://hal.science/hal-00678782}

Submitted on 13 Mar 2012

HAL is a multi-disciplinary open access archive for the deposit and dissemination of scientific research documents, whether they are published or not. The documents may come from teaching and research institutions in France or abroad, or from public or private research centers.
L'archive ouverte pluridisciplinaire HAL, est destinée au dépôt et à la diffusion de documents scientifiques de niveau recherche, publiés ou non, émanant des établissements d'enseignement et de recherche français ou étrangers, des laboratoires publics ou privés. 


\title{
PERFORMANCE EVALUATION OF POINT MATCHING METHODS IN VIDEO SEQUENCES WITH ABRUPT MOTIONS
}

\author{
Wael Elloumi*, Sylvie Treuillet, Remy Leconge \\ Institut Prisme, Polytech'Orléans, 12 rue de Blois, 45067 Orléans cedex2, France \\ wael.elloumi@univ-orleans.fr, Sylvie.treuillet@univ-orleans.fr, remy.leconge@univ-orleans.fr \\ Aicha Fonte \\ Institut Prisme, Polytech'Orléans, 12 rue de Blois, 45067 Orléans cedex2, France \\ aicha.fonte@univ-orleans.fr
}

\begin{abstract}
Keywords: Interest points, local descriptors, matching, performance evolution, video sequence, abrupt motions.
Abstract: In this paper, we compare the performance of matching algorithms in terms of efficiency, robustness, and computation time. Our evaluation uses as criterion, for efficiency and robustness, number of inliers and is carried out for different video sequences with abrupt motions (translation, rotation, combined). We compare SIFT, SURF, cross-correlation with Harris detector, and cross-correlation with SURF detector. Our experiments show that abrupt movements perturb a lot the matching process. They show also that SURF is the most disturbed, by such motions, and which even fails in cases that present a large rotation unlike the rest of descriptors as SIFT and cross-correlation.
\end{abstract}

\section{INTRODUCTION}

While the problem of image matching has been studied extensively for various applications, the remaining questions are which feature detector and descriptor performs the best and which one is the most appropriate to match images in real time for camera motion estimation. The field of our interest is the indoor/outdoor navigation assistance for the visually impaired with a low cost body mounted camera. Since vision based localization of mobile robots can rely on the assumption of the smooth movements, human motions are rougher and unpredictable and may cause loss in vision tracking. It will be wise to investigate the limits of the vision based localization especially in video sequences with abrupt motions.

Some previous works propose comparative studies of descriptors. Carneiro and Jepson (Carneiro and Jepson, 2002) introduce a phase-based local feature using Harris corner detector and compare it to the differential invariant features. They use the Receiver Operating Characteristic (ROC) curves as performance criterion and demonstrate that differential invariants are not the best for common illumination changes and 2D rotation. A variant of SIFT algorithm (Lowe, 2004) based PCA performs better on artificial data according to the recallprecision criterion (Ke and Sukthankar, 2004). Another extension of SIFT outperforms many local descriptors but is more costly in computation time (Mikolajczyk and Schmid, 2005).

All the references cited above compare descriptors on image pairs using data set with artificial or real geometric and photometric transformations but not on video sequences. In this paper, we propose a performance comparison of three popular point matching methods. SIFT (Lowe, 2004), SURF (Bay and al., 2006), and Harris (Harris and Stephen, 1988) with cross-correlation. All of these algorithms are evaluated in efficiency, robustness, and computation time criterion, using the same scenario. The comparison is based on sequences acquired with a camera attached to a robot hand. Efficiency and robustness is evaluated by the number of inliers (correct matches between two images). Next section presents the experimental setup, before results in section 3 and conclusion.

\footnotetext{
* This study is supported by HERON Technologies SAS and the Conseil Général du LOIRET.
} 


\section{EXPERIMENTAL SETUP}

Our experimental prototype is composed of USB PC camera $(320 \times 240$ pixels $)$ fixed on the gripper of a 6dof robot arm and which are connected to a desktop. Intrinsic parameters of the camera are estimated by a prior calibration. The robot arm can be controlled manually by its remote controller or automatically by programming dedicated software, using Cartesian or joint coordinate systems, with an adjustable velocity. This prototype allows us to capture video sequences with rotations, translations and combined motions including zoom effect and abrupt motions.

Experiments data set consists of nine video sequences acquired at $30 \mathrm{fps}$ frame rate in a real scene with brightness changes. We have chosen rotation, translation in the $\mathrm{y}$-direction to create a zoom effect, and combined motion with rotation and translation, to compare the different operators, because these kinds of motions are the most disturbing for matching process. Table 1 give details on the video sequences related to 3 types of motion: number of frame, shift or rotation angle between frames. Several velocities of the robot arm were tested during acquisition: low, medium, or high velocity. Furthermore, to simulate more abrupt motions and considerable transformations, we matched distant key frames. Velocities of motions present in these video sequences are faster than normal motions of a human being. For example, the lowest velocity of translation is $45 \mathrm{~cm}$ per second and the lowest velocity of rotation 100 degrees per second.

Based on the state of art presented in the previous section, we have chosen to compare SIFT because it's the most robust, cross correlation with Harris corner detector because it's the fastest and SURF descriptor which is considered as a good compromise between computation time and robustness. To have well distributed Harris points, we have divided the images in buckets of size $15 \times 15$ pixels. The ZNCC correlation score is applied in $11 \times 11$ pixels ROI, with a minimum threshold of 0.8 . The cross correlation is used with Harris and also with SURF detector to highlight the influence of the detector on matching process.

For evaluation, we observe the robustness and the computation time. The most popular metrics for robustness are ROC and Recall-Precision curves. Both are based on the number of correct matches and the number of false matches obtained for an image pair. We use the total number of correct matches (inliers) and the percentage of inliers compared to the total number of matched points (inliers and outliers), described by the equation (1).

$$
\% \text { inliers }=\frac{\text { correctmatches }}{\text { correctmatches }+ \text { falsematches }}
$$

The number of correct matches and false matches is determined with Least Median of Square algorithm (Zhang, 1998) by estimating the fundamental matrix in the image pair. The maximum distance from point to epipolar line, beyond which the point is considered an outlier and is not used for computing the final fundamental matrix is equal to 1 pixel. The desirable level of confidence that the matrix is correct is equal to $99 \%$. The only constraint of this method is that we must have at least eight matched features. The computation of the two-view geometry requires that the matches originate from a $3 \mathrm{D}$ scene and that the motion is more than a pure rotation. That is respected as the camera is fixed slightly out of the rotation axis on the robot clip.

To develop our comparative study, we perform the following process for each video sequence:

1. Fix the number of frames to skip (frame jump) between images to match.

2. Extract distinctive features in images and match them using the different descriptors.

3. Select inliers from these candidates by estimating the fundamental matrix using LMedS method.

\section{RESULTS}

In this section, we present in Figure 1 and Table 2 an extract of the results for all carried experiments and discuss the performance of the tested descriptors.

\subsection{Image rotation}

Matching is tested between images with a rotation angle between 7 and 120 degrees by varying velocity and image jump. The number of inliers clearly decreases for higher rotation velocity. SIFT descriptor is the most robust to rotation followed by SURF, which fails in fast rotation. Harris based matching is more disturbed than SURF based detector.

\subsection{Image scale change}

Scale change is achieved by a translation up to 1370 $\mathrm{mm}$. All descriptors have a similar robustness, (\% of inliers), slightly lower for cross correlation. SURF presents the lowest number of inliers for all 
velocities. The number of inliers decreases when increasing velocity of robot arm but much less than for the rotation case.

\subsection{Combined motion}

Combined motion is performed by simultaneously rotating and translating the robot arm (between 4 and 92 degrees with $1370 \mathrm{~mm}$ shift). The performance of all operators is worse than for simple transformation. SIFT clearly outcomes other descriptors in number of inliers. SURF is better than cross correlation based descriptor only for low velocity (v25) and even fails for large rotation velocity. Rotation is more disturbing than scale changes.

\section{CONCLUSION}

An experimental comparison of the famous matching descriptors is proposed to identify the most appropriate to estimate camera motion. To be as close as possible to our application, we have used several real video sequences with abrupt motions (rotation, scale change, and combined). SIFT performs the best results in terms of number of inliers, but it can not be used for real time applications. SURF and cross correlation are worse than SIFT but can be improved in order to be applied for real time applications. SURF is interesting in the case of scale change. However, its performance becomes similar to the cross correlation in the case of large rotations. In our tests, the matching process is achieved around one second for the best in half VGA images. This matching time remains too high for localizing a person in real time with a body-mounted camera. To overcome this issue, we can use the GPU programming for additional speed up. We also plan to exploit the extra capabilities of the latest smart phones to improve performance. New smart phones contains fast camera which can be combined with accelerometer and a GPS receiver, and future devices will contain magnetic compasses and gyroscopes.

\section{REFERENCES}

Bay, H., Tuytelaars, T., Van Gool, L., 2006. SURF: Speeded Up Robust Features, ECCV.

Carneiro, G., Jepson, A.D., 2002. Phase-Based Local Features. ECCV, 282-296.

Harris, C., Stephens, M., 1988. A combined corner and edge detector. In Alvey Vision Conf., 147-151.

Ke, Y., Sukthankar, R., 2004. PCA-SIFT: A More Distinctive Representation for Local Image Descriptors. CVPR'04, vol. 2, 506-513.

Lowe, D., 2004. Distinctive Image Features from Scale-Invariant Keypoints. In Int. J. of Computer Vision, vol.2, 91-110.

Mikolajczyk, K., Schmid, C., 2005. A performance evaluation of local descriptors. In IEEE Trans. on PAMI, 27(10), 1615-1630.

Zhang, Z., 1998. Determining the Epipolar Geometry and its Uncertainty. A Review in International Journal of Computer Vision, volume $27, \mathrm{n}^{\circ} 2$, pages 161-198.

Table 1: Video sequences data set.

\begin{tabular}{|c|c|c|c|}
\hline Motion & Video sequences & Number of frames & Motion range per frame \\
\hline \multirow{3}{*}{$\begin{array}{c}\text { Translation (scale } \\
\text { change) }\end{array}$} & Translation_v25 & 90 frames & $15.22 \mathrm{~mm}$ \\
\cline { 2 - 4 } & Translation_v50 & 49 frames & $27.96 \mathrm{~mm}$ \\
\cline { 2 - 4 } & Translation_v100 & 32 frames & $42.81 \mathrm{~mm}$ \\
\hline \multirow{3}{*}{ Rotation } & Rotation180_v25 & 55 frames & 3.39 degrees \\
\cline { 2 - 4 } & Rotation180_v50 & 24 frames & 20 degrees \\
\cline { 2 - 4 } & Rotation180_v100 & 11 frames & $8.51 \mathrm{~mm}$ and 4 degrees \\
\hline \multirow{3}{*}{ Combined } & Combined_v25 & 47 frames & $14.81 \mathrm{~mm}$ and 7.2 degrees \\
\cline { 2 - 4 } & Combined_v50 & 27 frames & $28.57 \mathrm{~mm}$ and 15 degrees \\
\cline { 2 - 4 } & Combined_v100 & 14 frames & \\
\cline { 2 - 4 }
\end{tabular}



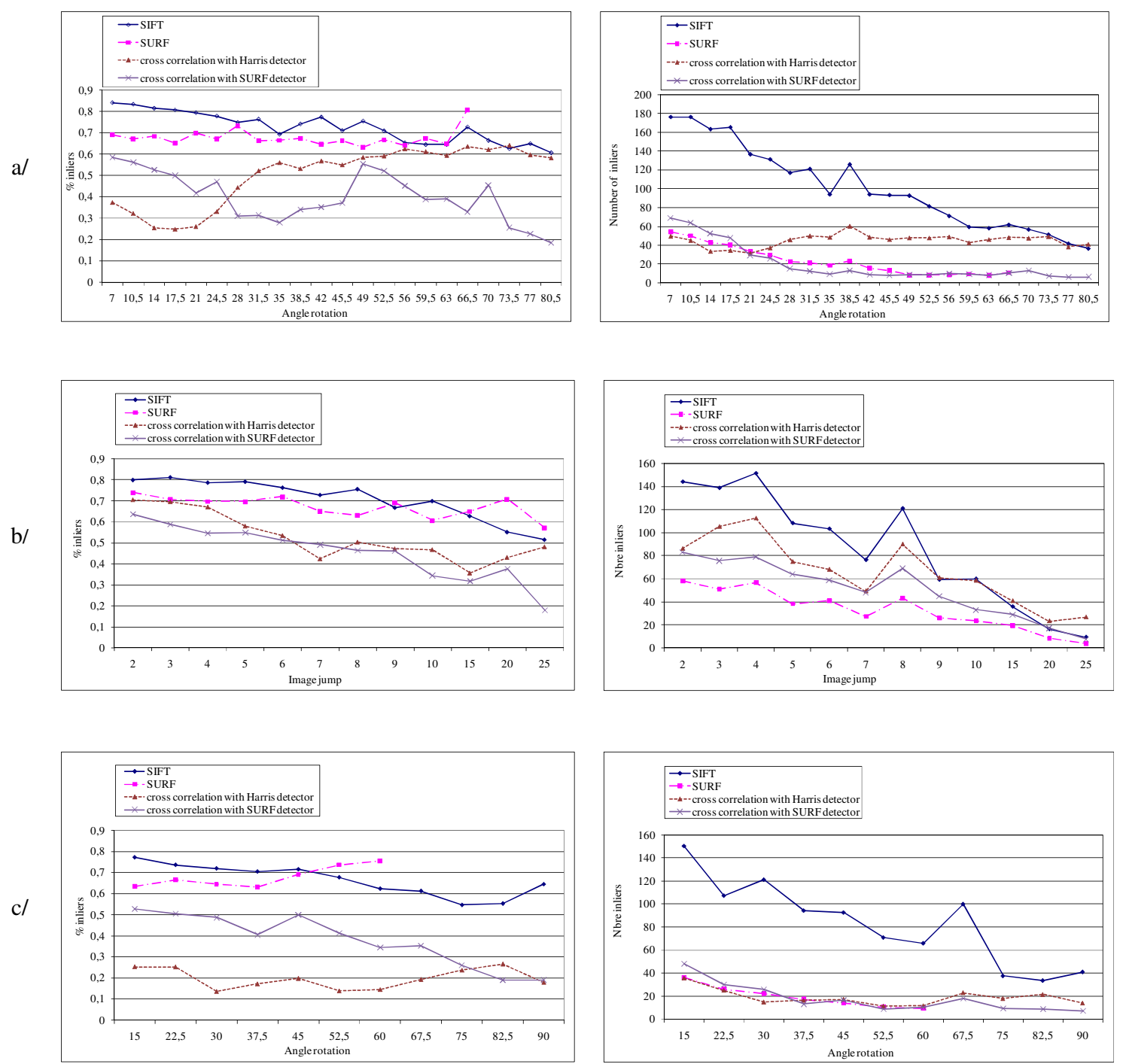

Figure 1: Evaluation of the robustness: $\%$ of inliers (left) and number of inliers (right) a/ Rotation180_v25 (first line) b/ Scaling Translation _v100 (second line) c/ Combined _v50 (third line)

Table 2: Computation time for an Intel dual core, $3 \mathrm{GHz}$, and 2GB memory (in milliseconds):

\begin{tabular}{|c|c|c|c|c|c|c|c|c|c|c|c|c|}
\cline { 2 - 14 } \multicolumn{1}{c|}{} & \multicolumn{3}{c|}{ SIFT } & \multicolumn{3}{c|}{ SURF } & \multicolumn{3}{c|}{$\begin{array}{c}\text { Cross correlation } \\
\text { with Harris }\end{array}$} & \multicolumn{3}{c|}{$\begin{array}{c}\text { Cross correlation } \\
\text { with SURF }\end{array}$} \\
\hline Datasets & Min & Max & Mean & Min & Max & Mean & Min & Max & Mean & Min & Max & Mean \\
\hline Trans_v25 & 1382 & 2065 & 1724 & 778 & 1411 & 1064 & 646 & 1817 & 918 & 718 & 1818 & 944 \\
\hline Rot180_v50 & 1433 & 2302 & 1706 & 610 & 1503 & 853 & 591 & 1229 & 888 & 564 & 1307 & 792 \\
\hline Comb_v100 & 1279 & 2354 & 1782 & 530 & 1732 & 886 & 604 & 1389 & 850 & 545 & 1327 & 814 \\
\hline
\end{tabular}

